\title{
COMMUNICATIONS
}

\section{[4+2]-Cycloaddition of Alkyl Vinyl Ethers to 3-Aroylpyrrolo[1,2-a]quinoxaline-1,2-dione}

\author{
P. S. Silaichev, M. A. Kryuchkova, and A. N. Maslivets \\ Perm State University, ul. Bukireva 15, Perm, 614990 Russia \\ e-mail:koh2@psu.ru
}

Received October 27, 2009

DOI: $10.1134 / \mathrm{S} 1070428010040330$

Reactions of 3-aroylpyrrolo[1,2-a]quinoxaline-1,2diones with activated alkenes were not studied previously. We examined reactions of 3-(4-chlorobenzoyl)4-(4-chlorophenyl)pyrrolo[1,2-a]quinoxaline-1,2-dione (I) with alkyl vinyl ethers IIa and IIb at a ratio of 1:5 in boiling toluene (20-30 min, until violet color typical of initial compound I disappeared) and obtained new difficultly accessible bridged heterocyclic compounds, 16-alkoxy-2,14-bis(4-chlorophenyl)-15-oxa-3,10-diazatetracyclo $\left[8 \cdot 7 \cdot 0 \cdot 0^{1,13} \cdot 0^{4,9}\right]$ heptadeca- $2,4,6,8,13-$ pentaene-11,12-diones IIIa and IIIb. Compounds IIIa and IIIb are likely to be formed via thermal [4+2]cycloaddition of the $\mathrm{O}=\mathrm{C}-\mathrm{C}^{3}=\mathrm{C}^{3 \mathrm{a}}$ conjugated bond system in pyrrolo[1,2-a]quinoxaline-1,2-dione $\mathbf{I}$ at the polarized double $\mathrm{C}=\mathrm{C}$ bond in vinyl ether IIa or IIb.

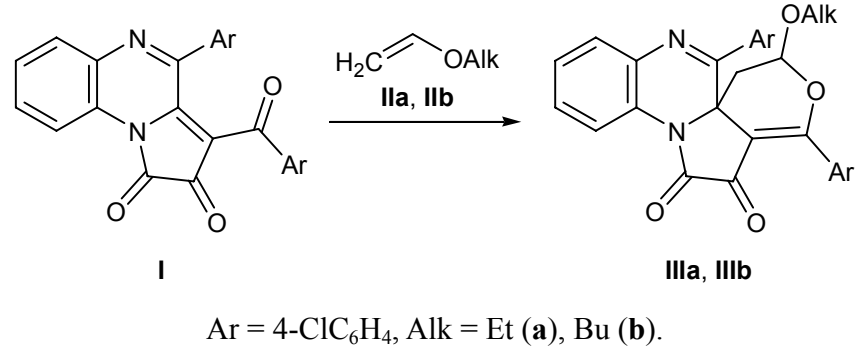

2,14-Bis(4-chlorophenyl)-16-ethoxy-15-oxa-3,10diazatetracyclo $\left[8.7 .0 .0^{1,13} \cdot 0^{4,9}\right]$ heptadeca-2,4,6,8,13pentaene-11,12-dione (IIIa). A solution of $5.0 \mathrm{mmol}$ of ethyl vinyl ether in $5 \mathrm{ml}$ of anhydrous toluene was added to a solution of $1.0 \mathrm{mmol}$ of 3-(4-chlorobenzoyl)-4-(4-chlorophenyl)pyrrolo[1,2-a]quinoxaline1,2-dione (I) in $50 \mathrm{ml}$ of anhydrous toluene. The mixture was heated for 20 min under reflux and cooled, the solvent was removed, and the precipitate was re- crystallized from toluene. Yield $78 \%, \mathrm{mp} 224-225^{\circ} \mathrm{C}$. IR spectrum, $v, \mathrm{~cm}^{-1}$ : $1728\left(\mathrm{C}^{11}=\mathrm{O}\right), 1713\left(\mathrm{C}^{12}=\mathrm{O}\right)$. ${ }^{1} \mathrm{H}$ NMR spectrum, $\delta$, ppm: $0.81 \mathrm{t}\left(3 \mathrm{H}, \mathrm{CH}_{3}, J=\right.$ $7.0 \mathrm{~Hz}), 2.18 \mathrm{~d} . \mathrm{d}(1 \mathrm{H}, 17-\mathrm{H}, J=13.4,4.6 \mathrm{~Hz}), 2.36 \mathrm{~d}$ $(1 \mathrm{H}, 17-\mathrm{H}, J=13.4 \mathrm{~Hz}), 3.37 \mathrm{~m}\left(1 \mathrm{H}, \mathrm{OCH}_{2}\right), 3.44 \mathrm{~m}$ $\left(1 \mathrm{H}, \mathrm{OCH}_{2}\right), 5.62 \mathrm{~d}(1 \mathrm{H}, 16-\mathrm{H}, J=4.6 \mathrm{~Hz}), 7.40$ $7.93 \mathrm{~m}\left(12 \mathrm{H}, \mathrm{H}_{\text {arom }}\right)$. Found, \%: C 65.75; H 4.38; N 5.15. $\mathrm{C}_{30} \mathrm{H}_{24} \mathrm{Cl}_{2} \mathrm{~N}_{2} \mathrm{O}_{4}$. Calculated, \%: C 65.82; $\mathrm{H} 4.42$; N 5.12 .

16-Butoxy-2,14-bis(4-chlorophenyl)-15-oxa-3,10diazatetracyclo $\left[8.7 .0 .0^{1,13} \cdot 0^{4,9}\right]$ heptadeca- $2,4,6,8,13-$ pentaene-11,12-dione (IIIb) was synthesized in a similar way from butyl vinyl ketone. Yield 74\%, mp 219$220^{\circ} \mathrm{C}$ (from ethyl acetate). IR spectrum, $v, \mathrm{~cm}^{-1}: 1726$ $\left(\mathrm{C}^{11}=\mathrm{O}\right), 1714\left(\mathrm{C}^{12}=\mathrm{O}\right) .{ }^{1} \mathrm{H}$ NMR spectrum, $\delta$, ppm: $0.72 \mathrm{t}\left(3 \mathrm{H}, \mathrm{CH}_{3}\right), 0.98 \mathrm{~m}\left(2 \mathrm{H}, \mathrm{CH}_{2}\right), 1.14 \mathrm{~m}\left(2 \mathrm{H}, \mathrm{CH}_{2}\right)$, 2.18 d.d $(1 \mathrm{H}, 17-\mathrm{H}, J=13.6,4.4 \mathrm{~Hz}), 2.35 \mathrm{~d}(1 \mathrm{H}$, $17-\mathrm{H}, J=13.6 \mathrm{~Hz}), 3.29 \mathrm{~m}\left(1 \mathrm{H}, \mathrm{OCH}_{2}\right), 3.39 \mathrm{~m}(1 \mathrm{H}$, $\left.\mathrm{OCH}_{2}\right), 5.60 \mathrm{~d}(1 \mathrm{H}, 16-\mathrm{H}, J=4.4 \mathrm{~Hz}), 7.38-7.90 \mathrm{~m}$ $\left(12 \mathrm{H}, \mathrm{H}_{\text {arom }}\right)$. Found, \%: C 65.75; H 4.38; N 5.15. $\mathrm{C}_{30} \mathrm{H}_{24} \mathrm{Cl}_{2} \mathrm{~N}_{2} \mathrm{O}_{4}$. Calculated, \%: C 65.82; $\mathrm{H} 4.42$; N 5.12.

The IR spectra were measured on a FSM-1201 spectrophotometer from samples dispersed in mineral oil. The ${ }^{1} \mathrm{H}$ NMR spectra were recorded on a Bruker AM-400 spectrometer at $400 \mathrm{MHz}$ using DMSO- $d_{6}$ as solvent and tetramethylsilane as internal reference. The purity of the isolated compounds was checked by TLC on Silufol plates using benzene-ethyl acetate $(5: 1)$ or ethyl acetate as eluent.

This study was performed under financial support by the Russian Foundation for Basic Research (project no. 08-03-01032). 